\title{
THE MINIMAL MODEL OF THE COMPLEMENT OF AN ARRANGEMENT OF HYPERPLANES
}

\author{
MICHAEL FALK
}

\begin{abstract}
In this paper the methods of rational homotopy theory are applied to a family of examples from singularity theory. Let $\mathbf{A}$ be a finite collection of hyperplanes in $\mathbf{C}^{l}$, and let $M=\mathbf{C}^{l}-\bigcup_{H \in \mathbf{A}} H$. We say $\mathbf{A}$ is a rational $K(\pi, 1)$ arrangement if the rational completion of $M$ is aspherical. For these arrangements an identity (the LCS formula) is established relating the lower central series of $\pi_{1}(M)$ to the cohomology of $M$. This identity was established by group-theoretic means for the class of fiber-type arrangements in previous work. We reproduce this result by showing that the class of rational $K(\pi, 1)$ arrangements contains all fiber-type arrangements. This class includes the reflection arrangements of types $A_{l}$ and $B_{l}$.

There is much interest in arrangements for which $M$ is a $K(\pi, 1)$ space. The methods developed here do not apply directly because $M$ is rarely a nilpotent space. We give examples of $K(\pi, 1)$ arrangements which are not rational $K(\pi, 1)$ for which the LCS formula fails, and $K(\pi, 1)$ arrangements which are not rational $K(\pi, 1)$ where the LCS formula holds. It remains an open question whether rational $K(\pi, 1)$ arrangements are necessarily $K(\pi, 1)$.
\end{abstract}

1. Introduction. An arrangement of hyperplanes is a finite collection of $\mathbf{C}$ linear subspaces of dimension $(l-1)$ in $\mathbf{C}^{l}$. To such an arrangement $\mathbf{A}$ is associated an open $2 l$-manifold, the complement $M=\mathbf{C}^{l}-\bigcup\{H \mid H \in \mathbf{A}\}$. The connections between the topology of $M$ and the combinatorial geometry of $\mathbf{A}$ are the source of much current research in this area. The most successful investigations concern the cohomology of $M[\mathbf{1 3}, \mathbf{1 5}]$, whereas the most difficult unsolved problems involve the homotopy groups of $M[\mathbf{5}]$. In this paper we study the link between cohomology and homotopy provided by Sullivan's theory of minimal models $[\mathbf{1 4}]$.

In [6], a numerical relationship was established between $\pi_{1}(M)$ and $H^{*}(M)$ for the class of fiber-type arrangements. This LCS formula reads as follows:

$$
\prod_{n \geq 1}\left(1-t^{n}\right)^{\varphi_{n}(M)}=P_{M}(-t)
$$

where the $\varphi_{n}(M)$ are the ranks of successive quotients in the lower central series of $\pi_{1}(M)$, and $P_{M}(t)$ is the Poincaré polynomial of $H^{*}(M)$. Because the sequence $\varphi_{n}(M)$ is related to the 1 -minimal model $\mathscr{S}$ of $M$, we conjecture in [5] that the LCS formula holds precisely when $\mathscr{S}$ determines $H^{*}(M)$. With the methods developed in this paper we can resolve this conjecture. Specifically, we show (Corollary 3.8) that the LCS formula holds when $H^{*}(\mathscr{S})$ is isomorphic to $H^{*}(M)$. Arrangements satisfying the latter condition are called rational $K(\pi, 1)$. Corollary 3.8 may be

Received by the editors June 1, 1987.

1980 Mathematics Subject Classification (1985 Revision). Primary 55P62, 57M05; Secondary $06 \mathrm{C} 10$. 
paraphrased as "rational $K(\pi, 1)$ implies LCS". We can also show (Example 5.3) that the converse is false. In $\S 4$, we show that the fiber-type arrangements of [6] are rational $K(\pi, 1)$, so that Corollary 3.8 implies our main result there.

Much work in this area has been done by T. Kohno. In [10] he obtained the LCS formula for the arrangements associated with the classical pure braid groups. This motivated our work in [6]. Recently he has proven the LCS formula for reflection arrangements of type $D_{l}[8]$, which are not fiber-type. And he has independently proven Corollary 3.8 [9]. The method of Kohno is more complicated because of its greater generality. The technique of this paper is simpler and more direct, being tailored specifically to complements of arrangements. In particular we exploit the formality (in the sense of [14]) of the complement $M$.

Our proof of 3.8 is based on the construction of the 1-minimal model $\mathscr{S}$ from the simple combinatorial model of $H^{*}(M)$ provided in [13]. The subsequent analysis of $\mathscr{S}$ greatly clarifies the relationship between the rational $K(\pi, 1)$ property and the LCS formula, and motivates the introduction of (algebraically) 2-determined arrangements (Definition 3.2). It is shown that rational $K(\pi, 1)$ arrangements are necessarily 2 -determined; the converse remains an open question.

The paper is organized as follows. In $\S 2$ we carry out the construction of the 1-minimal model of $M$, and obtain a useful decomposition into finite subcomplexes. The main results of the paper are stated and proved in $\S 3$. We give some examples in these sections to demonstrate the methods; in particular our approach yields a simple derivation of the recursive formulas for the $\varphi_{n}(M)$ which are implicit in the LCS formula. $\S 4$ contains the proof that fiber-type arrangements are rational $K(\pi, 1)$. This proof is independent of the rest of the paper. In $\S 5$ we give a necessary condition for 2-determined arrangements, and provide a counterexample for the converse of "rational $K(\pi, 1)$ implies LCS".

This research was undertaken in order to understand the higher homotopy in the complement of an arrangement. In particular there is much interest in arrangements for which $M$ is a $K(\pi, 1)$ space. There is clearly some relationship between the rational $K(\pi, 1)$ arrangements of this paper and topological $K(\pi, 1)$ arrangements (both classes contain all fiber-type arrangements), but the precise connection is unclear. The terminology "rational $K(\pi, 1)$ " is deceptive: a $K(\pi, 1)$ space is not necessarily rational $K(\pi, 1)$. The minimal model will in general give an accurate reflection of the fundamental group, and, in many cases, the higher homotopy groups as well. For instance, if $X$ is a $K(\pi, 1)$ space with nice fundamental group, the minimal model will reflect the asphericity of $X$. Such "nice" groups include nilpotent groups, free groups, and nilpotent extensions involving these groups. However, there are arrangements [5] with $K(\pi, 1)$ complements which are not rational $K(\pi, 1)$. It remains to be seen whether the results of this paper have any bearing on the various conjectures [5] concerning $K(\pi, 1)$ arrangements. In particular, the conjecture "rational $K(\pi, 1)$ implies $K(\pi, 1)$ " of [5] has not been resolved.

2. The 1-minimal model of $M$. We recall the elements of rational homotopy theory for non-simply-connected spaces. This material was compiled from various sources $[14,1,7,12]$.

Let $\mathbf{K}$ be a field of characteristic zero. A differential graded algebra (DG algebra) is a graded commutative algebra over $\mathbf{K}$ equipped with a (degree one) coboundary operator. Let $\left(\mathscr{A}^{*}, d\right)$ be a DG algebra. For each $n \geq 0$, let $\mathscr{A}(n)$ be the subalgebra 
generated by $\mathscr{A}^{k}, 0 \leq k \leq n$, and $d \mathscr{A}^{n}$. Construct subalgebras $\mathscr{A}(n, q)$ of $\mathscr{A}(n)$ for each $q \geq 0$ inductively as follows: $\mathscr{A}(n, 0)=\mathscr{A}(n-1)$, and, for $q>0, \mathscr{A}(n, q)$ is the subalgebra generated by $\mathscr{A}(n, q-1)$ and $\left\{x \in \mathscr{A}^{n} \mid d x \in \mathscr{A}(n, q-1)\right\}$.

DEFINITION 2.1 [1]. The DG algebra $\mathscr{M}$ is minimal provided

(i) $\mathscr{M}^{0} \cong \mathbf{K}$,

(ii) $\mathscr{M}$ is a free graded-commutative algebra, and

(iii) $\mathscr{M}(n)=\bigcup_{q \geq 0} \mathscr{M}(n, q)$ for each $n \geq 1$.

Given a $\mathbf{K}$-vector space $V$ of finite dimension, we denote by $\Lambda_{r}(V)$ the free graded-commutative algebra on $V$, with $V$ homogeneous of degree $r$. Thus $\Lambda_{r}(V)$ is an exterior or polynomial algebra depending on the parity of $r$.

Minimal algebras are usually built using the following construction.

DEFINITION $2.2[7]$. An inclusion $\mathscr{A} \subseteq \mathscr{B}$ of DG algebras is a Hirsch extension of degree $r$ if, for some $V, \mathscr{B} \cong \mathscr{A} \otimes \Lambda_{r}(V)$ as graded-commutative algebras, and $d V \subseteq \mathscr{A}^{r+1}$.

In practice, minimal algebras are constructed inductively by setting $\mathscr{M}(0,0)=$ $\mathscr{M}(0)=\mathbf{K}$ and $\mathscr{M}(n)=\bigcup_{q \geq 0} \mathscr{M}(n, q)$ for $n \geq 1$, where each $\mathscr{M}(n, q), q \geq 1$, is a degree $n$ Hirsch extension of $\mathscr{M}(n, q-1)$.

PROPOSITION $2.3\left[\mathbf{1}, 7.7\right.$ AND 7.8]. Let $\mathscr{A}$ be a $D G$ algebra with $H^{0}(\mathscr{A}) \cong$ K. Then there is a minimal algebra $\mathscr{M}$ and a $D G$ algebra map $f: \mathscr{M} \rightarrow \mathscr{A}$ such that $f^{*}: H^{*}(\mathscr{M}) \rightarrow H^{*}(\mathscr{A})$ is an isomorphism. The algebra $\mathscr{M}$ is unique up to isomorphism, and the map $f: \mathscr{M} \rightarrow \mathscr{A}$ is unique up to homotopy of DG algebra maps.

The algebra $\mathscr{M}$ of 2.3 is called the minimal model of $\mathscr{A}$. The subalgebra $\mathscr{M}(n)$ of $\mathscr{M}(n \geq 1)$ is called the $n$-minimal model of $\mathscr{A}$. The $n$-minimal model is characterized by the following properties:

(i) $\mathscr{M}(n)$ is a minimal algebra,

(ii) $\mathscr{M}(n)$ is generated by elements of degree at most $n$, and

(iii) $\left.f\right|_{\mathscr{M}(n)}: \mathscr{M}(n) \rightarrow \mathscr{A}$ induces isomorphisms in cohomology through degree $n$, and an injection in degree $(n+1)$.

(This is implied by $[\mathbf{1}, 7.9]$.) The minimal ( $n$-minimal) model of a connected simplicial complex $X$ is by definition the minimal ( $n$-minimal) model of the rational DG algebra of Q-polynomial forms on $X$. Our main tool will be the connection between the 1-minimal model of $X$ and the fundamental group of $X$. This connection is developed in the following paragraphs; a good reference is [12].

Let $\mathscr{A}$ be a DG algebra with $H^{0}(\mathscr{A})=\mathbf{K}$ and $H^{*}(\mathscr{A})$ finitely generated. In this case the 1-minimal model of $\mathscr{A}$ is an increasing union of degree one Hirsch extensions. Let $\mathscr{S}=\mathscr{M}(1)$ be the 1-minimal model, and set $\mathscr{S}(n)=\mathscr{M}(1, n)$. Then $\mathscr{S}(n-1) \subseteq \mathscr{S}(n)$ is a degree one Hirsch extension for each $n \geq 1$; write $\mathscr{S}(n) \cong \mathscr{S}(n-1) \otimes \Lambda_{1}\left(V_{n}\right)$. Sullivan [14] showed that the 1-minimal model $\mathscr{S}$ of a connected simplicial complex $X$ (with finitely generated rational cohomology) is dual to the Lie algebra determined by the rational nilpotent completion of the fundamental group of $X$. As a consequence, the vector spaces $V_{n}$ are related to the successive quotients in the lower central series of $\pi_{1}(X)$.

Let $G$ be a finitely presented group. Construct the lower central series $G_{n}$ of $G$ by setting $G_{0}=G$ and $G_{n}=\left[G_{n-1}, G\right]$ for $n \geq 1$. Set $G(n)=G_{n-1} / G_{n}$. 
PROPOSITION 2.4 (SULLIVAN $[\mathbf{1 4}, \mathbf{7}]$ ). Let $X$ be a connected simplicial complex with $H^{*}(X ; \mathbf{Q})$ finitely generated. Let $G=\pi_{1}(X, *)$ and $\mathscr{S}$ be the 1-minimal model of $X$. Then

$$
\operatorname{rank}(G(n))=\operatorname{dim} V_{n} .
$$

We denote $\operatorname{rank}(G(n))$ by $\varphi_{n}(X)$; these are the exponents appearing in the LCS formula.

The minimal model often contains information on the higher homotopy groups of a space. In particular, we point out another major result of Sullivan. Let $\pi^{n}(\mathscr{M})$ denote the vector space of indecomposable elements of degree $n$ in $\mathscr{M}$.

Proposition 2.5 (SUlLIVAN $[\mathbf{1 4}, \mathbf{7}]$ ). Let $X$ be a connected simplicial complex with $H^{*}(X: \mathbf{Q})$ finitely generated and $\pi_{1}(X, *)=1$. Let $\mathscr{M}$ be the minimal model of $X$. Then, for each $n \geq 2, \pi_{n}(X) \otimes \mathbf{Q}$ is isomorphic to $\operatorname{Hom}\left(\pi^{n}(\mathscr{M}), \mathbf{Q}\right)$.

This result can be generalized to nilpotent spaces $[\mathbf{1 4}, \mathbf{1}]$. For an arbitrary connected simplicial complex $X$, the minimal model is related to the $\mathbf{Q}$-completion of $X[\mathbf{2}]$; this fact is exploited in $\S 4$.

REMARK. In the sequel we will need to replace the DG algebra of Q-polynomial forms with the ordinary DeRham complex $A^{*}(M)$ of smooth forms on the manifold $M$. According to [7], the minimal model of the real DG algebra $A^{*}(M)$ is isomorphic to the minimal model of $M$, tensored with $\mathbf{R}$. Thus, real coefficients should be understood throughout the rest of this paper, except in $\S 4$. The reader should note that 2.4 remains valid with real coefficients.

We are now prepared to analyze the complement of an arrangement of hyperplanes.

Let $\mathbf{A}=\left\{H_{1}, \ldots, H_{n}\right\}$ be an arrangement in $\mathbf{C}^{l}$; each $H_{i}$ is a $\mathbf{C}$-linear subspace of dimension $(l-1)$ in $\mathbf{C}^{l}$. Let $M=\mathbf{C}^{l}-\bigcup_{i=1}^{n} H_{i}$ be the complement of $\mathbf{A}$. For each $i, 1 \leq i \leq n$, let $\omega_{i}=(1 / 2 \pi i) d\left(\log \phi_{i}\right)$, where $\phi_{i}$ is a linear form with kernel $H_{i}$. Each 1-form $\omega_{i}$ gives a 1 -form on $M$ by restriction. Let $\mathscr{R}$ denote the subalgebra of the DeRham complex generated over $\mathbf{R}$ by $\left\{\omega_{1}, \ldots, \omega_{n}\right\}$.

PROPOSITION 2.6 (BRIESKORN). The correspondence $\omega_{i} \rightarrow\left[\omega_{i}\right]$ induces an isomorphism of $\mathscr{R}$ with $H^{*}(M ; \mathbf{R})$.

This is proved in [3] for integer coefficients. Tensoring with $\mathbf{R}$ yields the corresponding result over the reals.

Proposition 2.6 implies that $M$ is a formal space, that is, that the real homotopy type of (the rational nilpotent completion of) $M$ is determined by $H^{*}(M)$. Indeed, by 2.6 the minimal model of $\mathscr{R}$ will be a minimal model for $A^{*}(M)$, and, again by 2.6, $\mathscr{R}$ is isomorphic to $H^{*}(M)$.

Let $\mathscr{E}=\Lambda_{1}\left(V_{1}\right)$, where $V_{1}$ is a vector space with basis $\left\{e_{1}, \ldots, e_{n}\right\}$ corresponding to the hyperplanes in $\mathbf{A}$. Set $d e_{i}=0$ for $1 \leq i \leq n$. If $J=\left\{i_{1}, \ldots, i_{p}\right\} \subseteq\{1, \ldots, n\}$, we write $e_{J}=e_{i_{1}} \wedge \cdots \wedge e_{i_{p}}$, and $\partial e_{J}=\sum_{k=1}^{p}(-1)^{k-1} e_{i_{1}} \wedge \cdots \wedge \bar{e}_{i_{k}} \wedge \cdots \wedge e_{i_{p}}$, where - indicates deletion. We say $J$ is dependent if the C-codimension of $H_{i_{1}} \cap \cdots \cap H_{i_{p}}$ is strictly less than $p$.

Let $\rho: \mathscr{E} \rightarrow \mathscr{R}$ be the DG algebra map which sends $e_{i}$ to $\omega_{i}$ for $1 \leq i \leq n$.

PROPOSITION 2.7 (ORLIK-SOLOMON). The map $\gamma: \mathscr{E} \rightarrow \mathscr{R}$ is surjective with kernel the ideal $\mathscr{I}$ generated by $\left\{\partial e_{J} \mid J \subseteq\{1, \ldots, n\}\right.$ is dependent $\}$. 
Proof. The first assertion is obvious. The second is established in [13] for complex coefficients; the proof carries through for real coefficients.

Propositions 2.6 and 2.7 together imply that the minimal model of $M$ is isomorphic to the minimal model of $\mathscr{E} / \mathscr{I}$. This complex has zero differential, so $H^{*}(\mathscr{E} / \mathscr{I}) \cong \mathscr{E} / \mathscr{I}$.

We will construct the 1-minimal model $\rho: \mathscr{S} \rightarrow \mathscr{E} / \mathscr{I}$ inductively. Set $\mathscr{S}(0)=$ $\mathbf{R}$ and $\mathscr{S}(1)=\mathscr{E}$, with $\rho: \mathscr{S}(1) \rightarrow \mathscr{E} / \mathscr{I}$ the natural projection. Assume $n \geq$ 2 and $\rho: \mathscr{S}(n-1) \rightarrow \mathscr{E} / \mathscr{I}$ has been constructed, satisfying $\rho \circ d=0$. Let $V_{n}$ be a vector space isomorphic to the kernel of the map $\rho^{*}: H^{2}(\mathscr{S}(n-1)) \rightarrow$ $(\mathscr{E} / \mathscr{I})^{2}$. Let $\mathscr{S}(n)$ be a degree one Hirsch extension of $\mathscr{S}(n-1)$ with $\mathscr{S}(n) \cong$ $\mathscr{S}(n-1) \otimes \Lambda_{1}\left(V_{n}\right)$ and $d: V_{n} \rightarrow \mathscr{S}(n-1)^{2}$ any linear choice of representatives; that is, the composite $V_{n} \rightarrow$ image $(d) \rightarrow H^{2}(\mathscr{S}(n-1))$ should be an isomorphism. The map $\rho: \mathscr{S}(n-1) \rightarrow \mathscr{E} / \mathscr{I}$ is extended to $\mathscr{S}(n)$ by setting $\rho(v)=0$ for $v \in V_{n}$. By construction of $d$, we have for any $v \in V_{n}$ that $\rho \circ d(v)$ is exact in $\mathscr{E} / \mathscr{I}$; since $\mathscr{E} / \mathscr{I}$ has zero differential, $\rho \circ d(v)=0$. It follows that $\rho \circ d=0$.

Let $\mathscr{S}=\bigcup_{n=0}^{\infty} \mathscr{S}(n)$, with $\rho: \mathscr{S} \rightarrow \mathscr{E} / \mathscr{I}$ as above. The condition $\rho \circ d=0$ guarantees that $\rho$ is a DG algebra map. The observations below will show that $\mathscr{S}$ is the 1 -minimal model of $\mathscr{E} / \mathscr{I}$. We set $V_{1}=\mathscr{E}$, so that $\mathscr{S}(1)=\Lambda_{1}\left(V_{1}\right)$. Note that $\left.d\right|_{V_{1}}=0$.

PROPOSITION 2.8. (i) $d V_{n} \cap \mathscr{S}(n-2)^{2}=0$ for $n \geq 2$.

(ii) The restriction $d: \bigoplus_{n \geq 2} V_{n} \rightarrow \mathscr{S}^{2}$ is injective.

PROOF. The second assertion follows easily from the first, since $d: V_{n} \rightarrow$ $\mathscr{S}(n-1)^{2}$ is injective for $n \geq 2$. For property (i), observe that $H^{2}(\mathscr{S}(n-2)) \rightarrow$ $H^{2}(\mathscr{S}(n-1))$ kills the kernel of $H^{2}(\mathscr{S}(n-2)) \rightarrow(\mathscr{E} / \mathscr{I})^{2}$. Since $V_{n} \rightarrow$ image $(d) \rightarrow H^{2}(\mathscr{S}(n-1))$ is injective, the result follows.

Corollary 2.9. The map $\rho: \mathscr{S} \rightarrow \mathscr{E} / \mathscr{I}$ is the 1-minimal model.

PROOF. The DG algebra $\mathscr{S}$ is clearly minimal, and generated in degree one. From 2.8(ii), we conclude that $H^{1}(\mathscr{S}) \cong H^{1}(\mathscr{S}(1)) \cong V_{1}$, so that $\rho^{*}: H^{1}(\mathscr{S}) \rightarrow$ $(\mathscr{E} / \mathscr{I})^{1}$ is an isomorphism. That $\rho^{*}: H^{2}(\mathscr{S}) \rightarrow(\mathscr{E} / \mathscr{I})^{2}$ is injective follows from our previous observation that the kernel of $H^{2}(\mathscr{S}(n)) \rightarrow(\mathscr{E} / \mathscr{I})^{2}$ vanishes in $H^{2}(\mathscr{S}(n+1))$.

PROPOSITION 2.10. The map $\rho^{*}: H^{*}(\mathscr{S}) \rightarrow \mathscr{E} / \mathscr{I}$ is surjective. In particular, $\rho^{*}: H^{2}(\mathscr{S}) \rightarrow(\mathscr{E} / \mathscr{I})^{2}$ is an isomorphism.

COROllaRY 2.11. The map $\rho: \mathscr{S} \rightarrow \mathscr{E} / \mathscr{I}$ is the minimal model if and only if $\rho^{*}$ is injective.

Let us analyze the construction of $\mathscr{S}$ in more detail. First observe that $V_{2}$ is isomorphic to $\mathscr{J}^{2}$, the degree two part of the relation ideal. In fact, $d: V_{2} \rightarrow \mathscr{S}(1)^{2}$ is an isomorphism onto $\mathscr{J}^{2}$. By definition of $\mathscr{I}$, the subspace $\mathscr{I}^{2}$ has a basis consisting of elements of the form $e_{q} e_{r}-e_{p} e_{r}+e_{p} e_{q}=\left(e_{p}-e_{r}\right)\left(e_{q}-e_{r}\right)$, where $H_{p} \cap H_{q} \cap H_{r}$ has codimension two $\left(H_{p}, H_{q}, H_{r} \in \mathbf{A}\right)$.

PROPOSITION 2.12. Suppose $V_{n}=0$ for some $n \geq 2$. Then $V_{n}=0$ for all $n \geq 2$, and the hyperplanes of $\mathbf{A}$ are in general position through codimension two.

ProOF. If $H_{p}, H_{q}, H_{r} \in \mathbf{A}$ with $H_{p} \cap H_{q} \cap H_{r}$ having codimension two, then there is a $v_{2} \in V_{2}$ with $d v_{2}=\left(e_{p}-e_{r}\right)\left(e_{q}-e_{r}\right)$. Then $d\left(\left(e_{p}-e_{r}\right) v_{2}\right)=0$ and 
$\rho\left(\left(e_{p}-e_{r}\right) v_{2}\right)=0$, so there is a $v_{3} \in V_{3}$ with $d v_{3}=\left(e_{p}-e_{r}\right) v_{2}$. Continuing, we obtain a sequence $v_{n}, n \geq 2$, with $v_{n} \in V_{n}$ and $d v_{n+1}=\left(e_{p}-e_{r}\right) v_{n}$. Thus $V_{n} \neq 0$ for all $n \geq 2$. The result follows.

This last result, together with 2.4 , imply that $\pi_{1}(M)$ is not nilpotent unless $\mathbf{A}$ is general position through codimension two, in which case $\pi_{1}(M)$ is known to be abelian.

The dimension of $V_{2}$ is easy to compute

PROPOSITION 2.13. $\operatorname{dim} V_{2}=\left(\begin{array}{c}n \\ 2\end{array}\right)-\operatorname{dim} H^{2}(M)$, where $n$ is the number of hyperplanes in $\mathbf{A}$.

PROOF.

$$
\begin{aligned}
\operatorname{dim}\left(V_{2}\right) & =\operatorname{dim}\left(\mathscr{J}^{2}\right)=\operatorname{dim}\left(\mathscr{E}^{2}\right)-\operatorname{dim}\left((\mathscr{E} / \mathscr{J})^{2}\right) \\
& =\left(\begin{array}{l}
n \\
2
\end{array}\right)-\operatorname{dim}\left(H^{2}(M)\right) .
\end{aligned}
$$

The betti number $\operatorname{dim} H^{2}(M)$ is easily computed using Möbius functions as in [13].

Now, the algebra $\mathscr{S}$ is a free exterior algebra on $\bigoplus_{n>1} V_{n}$. We define a graded subspace $U_{k}, 0 \leq k<\infty$, consisting of elements of $\mathscr{S}^{-}$with index sum $k$. More precisely, $U_{k}^{p}=\bigoplus V_{i_{1}} \wedge \cdots \wedge V_{i_{p}}$, summing over all $\left\{i_{1}, \ldots, i_{p}\right\}$ satisfying $1 \leq i_{1} \leq$ $\cdots \leq i_{p}$ with $i_{1}+\cdots+i_{p}=k$. By convention, $U_{0}^{0}=\mathscr{S}^{0}=\mathbf{R}$, and $U_{0}^{p}=0$ for $p \geq 1$.

Proposition 2.14. (i) $\mathscr{S}=\bigoplus_{k \geq 0} U_{k}$.

(ii) $U_{k}^{p}=0$ if $p>k$,

(iii) $U_{k}^{1}=V_{k}$,

(iv) $U_{k}^{k} \cong \mathscr{E}^{k}$, and

(v) $U_{k}^{k-1} \cong \mathscr{E}^{k-2} \otimes V_{2}$.

PROOF. The proofs are all straightforward and will be left to the reader.

The crucial observation is that $d$ preserves index sum, so that each $U_{k}$ is a subcomplex. We establish this result in the next sequence of propositions.

PROPOSITION 2.15. $d V_{n} \subseteq U_{n}^{2} \cap \mathscr{S}(n-1)^{2}$.

PROOF. We argue by induction on $n$. The case $n=2$ is verified in the discussion preceding 2.12.

Assume $d V_{k} \subseteq U_{k}^{2} \cap \mathscr{S}(k-1)^{2}$ for all $k<n$. We claim that $d\left(U_{k}^{2} \cap \mathscr{S}(n-1)^{2}\right) \subseteq$ $U_{k}^{3}$ for any $k$. To see this, observe that, if $x \in V_{p}$ and $y \in V_{q}$ with $d x \in U_{p}^{2}$ and $d y \in U_{q}^{2}$, then $d(x y) \in U_{p+q}^{3}$. Since any element of $U_{k}^{2} \cap \mathscr{S}(n-1)^{2}$ is a sum of terms $x y$ with $x \in V_{p}, y \in V_{q}$ satisfying $p+q=k$ and $p, q \leq n-1$, the claim follows from our inductive assumption.

Now choose a basis for $V_{n}$, and let $v$ be a basis element. The choice of $d$ in the construction of $\mathscr{S}$ allows the alteration of $d v$ by a coboundary in $\mathscr{S}(n-1)^{2}$. This is done in such a way that $d v$ will lie in $U_{n}^{2}$. Using $2.14(\mathrm{i})$, we write $d v=u_{2}+\cdots+u_{m}$ with $u_{i} \in U_{i}^{2}$ for $2 \leq i \leq m$. According to $2.14(\mathrm{i})$ and the claim above, we must have $d u_{i}=0$ for each $i$. Note that $\rho\left(u_{i}\right)=0$ for $3 \leq i \leq m$, by definition of $\rho$, and $\rho\left(u_{2}\right)=\rho(d v)=0$ also. Thus any $u_{i}$ which lies in $\overline{\mathscr{S}}(n-2)^{2}$ is exact in $\mathscr{S}(n-1)^{2}$ and may be eliminated. This is certainly the case for all $i<n$. So we may assume $i>n$ and $u_{i}=x+y$ with $x \in U_{i}^{2} \cap \mathscr{S}(n-2)^{2}$ and $y \in \bigoplus_{p=2}^{n-1} V_{p} \wedge V_{n-1}$. Using 
$d x+d y=0$, Proposition 2.8(ii), and standard facts about linear independence in tensor products, it can be shown that $y=0$. The calculation is tedious but straightforward and will be left to the reader. As a result, $u_{i}=x \in \mathscr{S}(n-2)^{2}$, and so $u_{i}$ may be eliminated. Thus $d v=u_{n} \in U_{n}^{2} \cap \mathscr{S}(n-1)^{2}$. This completes the inductive step, and the result follows.

The general result is an easy consequence of 2.15 .

PROPOSITION 2.16. $d U_{k}^{p} \subseteq U_{k}^{p+1}$.

PROOF. Let $1 \leq i_{1} \leq \cdots \leq i_{p}$ with $i_{1}+\cdots+i_{p}=k$. Now

$$
d\left(V_{i_{1}} \wedge \cdots \wedge V_{i_{p}}\right) \subseteq \sum_{j=1}^{p} V_{i_{1}} \wedge \cdots \wedge d V_{i_{j}} \wedge \cdots \wedge V_{i_{p}} .
$$

By $2.15, d V_{i_{j}} \subseteq U_{i_{j}}^{2}$. Since $V_{i_{1}} \wedge \cdots \wedge U_{i_{j}}^{2} \wedge \cdots \wedge V_{i_{p}} \subseteq U_{k}^{p+1}$ for each $j$, the assertion follows.

COROLLARY 2.17. $H^{*}(\mathscr{S}) \cong \bigoplus_{k \geq 0} H^{*}\left(U_{k}\right)$.

We have succeeded in decomposing the 1-minimal model $\mathscr{S}$ as a direct sum of the subcomplexes $U_{k}$. By 2.14(ii), each $U_{k}$ is a finite complex

$$
0 \rightarrow U_{k}^{1} \rightarrow \cdots \rightarrow U_{k}^{k} \rightarrow 0 .
$$

This observation yields the main results in $\S 3$, and also facilitates the computation of the $\varphi_{n}(M)$. The calculation is manageable for arrangements of seven hyperplanes or less and small values of $n$.

PROPOSITION 2.18. For $n \geq 3, \varphi_{n}(M)$ is equal to the kernel rank of the linear map $d: U_{n}^{2} \rightarrow U_{n}^{3}$.

PROOF. For $n>k$, each summand of $U_{n}^{k}$ has a factor of $V_{i}, i \geq 2$. Since $\left.\rho\right|_{V_{i}}=0$ for $i \geq 2$, we have $\left.\rho\right|_{U_{n}^{k}}=0$ for $n>k$. Since $\rho^{*}: H^{k}(\mathscr{S}) \rightarrow(\mathscr{E} / \mathscr{I})^{k}$ is injective for $k=1,2$, we have $H^{1}\left(U_{n}\right)=0=H^{2}\left(U_{n}\right)$ for $n \geq 3$. Then the sequence $0 \rightarrow U_{n}^{1} \rightarrow U_{n}^{2} \rightarrow U_{n}^{3}$ is exact. Since $U_{n}^{1}=V_{n}$, and $\varphi_{n}(M)=\operatorname{dim} V_{n}$ by 2.4 , the result follows easily.

According to $2.13, \varphi_{2}(M)=\left(\begin{array}{c}b_{1} \\ 2\end{array}\right)-b_{2}$, where $b_{p}=\operatorname{rank} H^{p}(M)$ is the $p$ th betti number of $M$. Clearly $\varphi_{1}(M)=b_{1}$, the number of hyperplanes in $\mathbf{A}$.

EXAMPLE 2.19. Let $\mathbf{A}$ be the arrangement in $\mathbf{C}^{3}$ consisting of the seven hyperplanes $x= \pm y, x= \pm z, y= \pm z$, and $z=0$. This is a free, simplicial arrangement with many interesting properties (see [5] for a detailed discussion). The betti numbers of the complement are $b_{0}=1, b_{1}=7, b_{2}=15$, and $b_{3}=9$. Thus $\varphi_{1}(M)=\operatorname{dim} V_{1}=7$, and $\varphi_{2}(M)=\operatorname{dim} V_{2}=\left(\begin{array}{l}7 \\ 2\end{array}\right)-15=6$. The degree two part $\mathscr{J}^{2}$ of the relation ideal has a basis arising from the six 3-fold intersections in codimension two. Using this basis, the map $d: V_{2} \rightarrow U_{2}^{2}=\mathscr{E}^{2}$ is defined (see remarks following 2.11). Using this, one writes the matrix of $d: U_{3}^{2} \rightarrow U_{3}^{3}$. By 2.14, this is a map from $\mathscr{E}^{1} \otimes V_{2}$ into $\mathscr{E}^{3} ;$ it is equivalent to the map $\mathscr{E}^{1} \otimes \mathscr{J}^{2} \rightarrow \mathscr{E}^{3}$ which sends $x \otimes y$ to $x y$. The size of this matrix is $\left(\begin{array}{l}7 \\ 3\end{array}\right)=35$ by $(7)(6)=42$. One must row reduce to find a basis for the kernel. This provides the rank $\varphi_{3}(M)$, and is also used to define $d: V_{3} \rightarrow U_{3}^{(2)}$, which is needed for the computation of $\varphi_{4}(M)$. As in the proof of 2.12 , each basis element of $\mathscr{J}^{2}$ gives rise to two elements of 
$\mathscr{E}^{1} \otimes V_{2}$ which are killed by $d$. Thus $\varphi_{3}(M) \geq 12$. This observation also reduces the problem to the row-reduction of a 35 by 30 matrix. The enterprising reader will find that $\varphi_{3}=17$.

The computation of $\varphi_{4}(M)$ is already too large to be done by hand. One must examine $d: U_{4}^{2} \rightarrow U_{4}^{3}$, which maps $U_{4}^{2}=V_{1} \wedge V_{3} \oplus \Lambda^{2}\left(V_{2}\right)$ into $U_{4}^{3}=\Lambda^{2}\left(V_{1}\right) \wedge V_{2}$. The size of this matrix is $\left(\begin{array}{l}7 \\ 2\end{array}\right) \cdot 6=126$ by $(7)(17)+\left(\begin{array}{l}6 \\ 2\end{array}\right)=134$. As above, one easily finds three independent kernel elements for each basis element of $\mathscr{I}^{2}$; this reduces the size of the computation to 126 by 116 .

We will return to this example throughout the remainder of the paper.

3. Rational $K(\pi, 1)$ arrangements and the LCS formal. In this section we apply the results of $\S 2$ to derive equivalent conditions for rational $K(\pi, 1)$ arrangements (Corollary 3.5) and for arrangements satisfying the LCS formula (Corollary 3.7). As a corollary we obtain the result "rational $K(\pi, 1)$ implies LCS". We preserve the notation of $\S 2$.

DEFINITION 3.1. The arrangement $\mathbf{A}$ is a rational $K(\pi, 1)$ if $\rho^{*}: H^{*}(\mathscr{S}) \rightarrow$ $\mathscr{E} / \mathscr{I}$ is an isomorphism.

So, if $\mathbf{A}$ is a rational $K(\pi, 1)$, then $\mathscr{S}$ is the minimal model of $A^{*}(M)$. By 2.11, this holds when $\rho^{*}$ is injective. The terminology is motivated by 2.5. (The minimal model of $M$ will satisfy $\pi^{n}(\mathscr{M})=0$ for $n \geq 2$.) However, as pointed out in $\S 2$, $M$ will be a nilpotent space only in trivial situations, so the general version of 2.5 rarely applies in our setting. In fact, the only rational $K(\pi, 1)$ arrangements for which $M$ is nilpotent are the arrangements of coordinate hyperplanes in $\mathbf{C}^{l}, l \geq 1$. In general, $\mathbf{A}$ is a rational $K(\pi, 1)$ if and only if the rational nilpotent completion of $M$ is aspherical (see $\S 4$ ).

Let $\mathcal{J} \subset \mathscr{E}$ be the ideal generated by $\mathscr{J}^{2}$.

DEFINITION 3.2. The arrangement $\mathbf{A}$ is (algebraically) 2-determined if $\mathscr{J}=\mathscr{I}$.

In 2-determined arrangements, all relations in $H^{*}(M)$ arise from codimension two degeneracies. This should be compared with the notion of formal arrangements $[5,2.7 .11]$, which might be called "geometrically 2-determined". Definition 3.2 generalizes the notion of parallel for arrangements in $\mathbf{C}^{3}[\mathbf{5}, 2.7 .10]$. That is, parallel arrangements in $\mathbf{C}^{3}$ are 2-determined.

We proceed to analyze $\rho^{*}: H^{*}(\mathscr{S}) \rightarrow \mathscr{E} / \mathscr{I}$ using the decomposition of Corollary 2.17 .

PROPOSITION 3.3. $H^{n}\left(U_{n}\right)=(\mathscr{E} / \mathscr{J})^{n}$.

ProOF. To compute $H^{n}\left(U_{n}\right)$, consider the sequence $U_{n}^{n-1} \rightarrow U_{n}^{n} \rightarrow 0$. We have $U_{n}^{n-1}=\mathscr{E}^{n-2} \otimes V_{2}$ and $U_{n}^{n}=\mathscr{E}^{n}$ by 2.14 , and $H^{n}\left(U_{n}\right)=\mathscr{E}^{n} /$ image $(d)$. As was noted in remarks following $2.11,\left.d\right|_{V_{2}}$ is an isomorphism onto $\mathscr{I}^{2} ;\left.d\right|_{\mathscr{E}}$ is identically zero. It follows that $d\left(\mathscr{E}^{n-2} \otimes V_{2}\right)=\mathscr{J}^{n}$. Therefore $H^{n}\left(U_{n}\right)=$ $\mathscr{E}^{n} / \mathscr{J}^{n}=(\mathscr{E} / \mathscr{J})^{n}$.

PROPOSITION 3.4. The kernel of $\rho^{*}: H^{n}(\mathscr{S}) \rightarrow(\mathscr{E} / \mathscr{I})^{n}$ is precisely $\bigoplus_{p>n} H^{n}\left(U_{p}\right) \oplus(\mathscr{I} / \mathscr{J})^{n}$.

ProOF. As noted in the proof of 2.18, $\rho$ is identically zero on $U_{p}^{n}$ for $p>n$. When $p<n, U_{p}^{n}=0$ by 2.14. And $\rho^{*}: H^{n}\left(U_{n}\right) \rightarrow(\mathscr{E} / \mathscr{I})^{n}$ is equivalent by 3.3 to the projection $(\mathscr{E} / \mathscr{J})^{n} \rightarrow(\mathscr{E} / \mathscr{J})^{n}$. The assertion is then a result of the decomposition $H^{n}(\mathscr{S})=\bigoplus_{p \geq 0} H^{n}\left(U_{p}\right)$ of 2.17 . 
COROLlaRY 3.5. The arrangement $\mathbf{A}$ is a rational $K(\pi, 1)$ if and only if $\mathbf{A}$ is 2-determined and $H^{n}\left(U_{p}\right)=0$ for all $0 \leq n<p$.

This last result is of limited utility because of the latter condition, which is false in general and virtually impossible to check with particular arrangements. There is some evidence to support the conjecture "rational $K(\pi, 1)$ if and only if 2-determined".

We now discuss the implications of $\S 2$ with respect to the LCS formula. Let $G=\pi_{1}(M)$ and let $G=G_{0} \supseteq G_{1} \supseteq \cdots \supseteq G_{n} \supseteq \cdots$ be the lower central series of $G$ as defined in $\S 2$. Let $\varphi_{n}$ be the rank of $G_{n-1} / G_{n}$ for $n \geq 1$. Let $b_{p}$ be the $p$ th betti number of $M$. Let $P(t)=\sum_{p \geq 0} b_{p} t^{p}$, the Poincaré polynomial of $M$. The following identity holds for a wide class of arrangements [6].

LCS formula.

$$
\prod_{n \geq 1}\left(1-t^{n}\right)^{\varphi_{n}}=P(-t) .
$$

For example, when $\mathbf{A}$ consists of $(r+1)$ lines through the origin in $\mathbf{C}^{2}, \pi_{1}(M)$ is the product of a free group of rank $r$ and an infinite cyclic group. In this case the LCS formula is Witt's formula [11, p. 330] for free groups, multiplied by $(1-t)$.

By 2.4, $\varphi_{n}=\operatorname{dim} V_{n}$. So the left-hand side of $(*)$ bears some relation to the 1 -minimal model $\mathscr{S}$. The connection is made precise using the decomposition $\mathscr{S}=\bigoplus_{k \geq 0} U_{k}$. We introduce a formal power series $Q(t)$ defined by

$$
Q(t)=\sum_{p \geq 0} \chi\left(U_{p}\right) t^{p}
$$

where $\chi\left(U_{p}\right)$ is the Euler characteristic of the finite complex $U_{p}$.

Proposition 3.6. Let $\mathbf{A}$ be an arbitrary arrangement. Then

$$
\prod_{n \geq 1}\left(1-t^{n}\right)^{\varphi_{n}}=Q(t)
$$

We will postpone the proof of 3.6 to the end of this section.

COROLLARY 3.7. The arrangement $\mathbf{A}$ satisfies the LCS formula if and only if $\chi\left(U_{p}\right)=(-1)^{p} b_{p}$ for all $p \geq 0$.

ProOF. Comparing coefficients in $Q(t)$ and $P(-t)$, the result is obvious.

COROllary 3.8. Suppose $\mathbf{A}$ is a rational $K(\pi, 1)$ arrangement. Then $\mathbf{A}$ satisfies the LCS formula.

Proof. According to $3.5, H^{n}\left(U_{p}\right)=0$ for $n<p$. Since $U_{p}^{n}=0$ for $n>p, 2.17$ implies $H^{p}\left(U_{p}\right)=H^{p}(\mathscr{S})$, which is isomorphic to $(\mathscr{E} / \mathcal{I})^{p}$ by hypothesis. Thus

$$
\chi\left(U_{p}\right)=\chi\left(H^{*}\left(U_{p}\right)\right)=(-1)^{p} \operatorname{rank} H^{p}\left(U_{p}\right)=(-1)^{p} b_{p} .
$$

The result now follows from 3.7.

The converse of 3.8 is false; a counterexample will be furnished in $\S 5$. (This example also shows that the latter condition of Corollary 3.5 is not satisfied in general.)

Corollary 3.7 provides an easy method for determining the recursive formulas among the $\varphi_{n}$ which are implicit in the LCS formula. We demonstrate by deriving 
the formulas for $\varphi_{3}$ and $\varphi_{4}$. Recall that $\varphi_{1}=b_{1}$ and $\varphi_{2}=\left(\begin{array}{c}b_{1} \\ 2\end{array}\right)-b_{2}$ are satisfied in general. Now assume $\mathbf{A}$ satisfies the LCS formula. We compute the Euler characteristic of the chain complex $0 \rightarrow U_{3}^{1} \rightarrow U_{3}^{2} \rightarrow U_{3}^{3} \rightarrow 0$, using $U_{3}^{1}=V_{3}$, $U_{3}^{2}=V_{1} \wedge V_{2}$, and $U_{3}^{3}=\Lambda^{3}\left(V_{1}\right)$. Thus $\chi\left(U_{3}\right)=-\varphi_{3}+\varphi_{1} \varphi_{2}-\left(\begin{array}{c}\varphi_{1} \\ 3\end{array}\right)$, and 3.7 yields

$$
-b_{3}=-\varphi_{3}+\varphi_{1} \varphi_{2}-\left(\begin{array}{c}
\varphi_{1} \\
3
\end{array}\right) \text {. }
$$

Similarly, the computation of $\chi\left(U_{4}\right)$, using $U_{4}^{1}=V_{4}, U_{4}^{2}=V_{1} \wedge V_{3} \oplus \Lambda^{2}\left(V_{2}\right)$, $U_{4}^{3}=\Lambda^{2}\left(V_{1}\right) \wedge V_{2}$, and $U_{4}^{4}=\Lambda^{4}\left(V_{1}\right)$, yields the formula

$$
b_{4}=-\varphi_{4}+\varphi_{1} \varphi_{3}+\left(\begin{array}{c}
\varphi_{2} \\
2
\end{array}\right)-\left(\begin{array}{c}
\varphi_{1} \\
2
\end{array}\right) \varphi_{2}+\left(\begin{array}{c}
\varphi_{1} \\
4
\end{array}\right) .
$$

Let us check the $\varphi_{3}$ formula for the arrangement of Example 2.19. Recall that $b_{3}=9, \varphi_{1}=7$, and $\varphi_{2}=6$. The formula for $\varphi_{3}$ above yields $-9=-\varphi_{3}+42-35$, or $\varphi_{3}=16$. Since we computed $\varphi_{3}=17$, we conclude that this arrangement does not satisfy the LCS formula. This is a $K(\pi, 1)$ arrangement, since it is simplicial [4]. However, by 3.8 it is not a rational $K(\pi, 1)$.

We now prove 3.6. The Poincaré series of a bigraded module $C=\bigoplus_{i, j \geq 0} C_{i, j}$ is defined by $P_{C}(x, y)=\sum_{i, j \geq 0}\left(\operatorname{dim} C_{i, j}\right) x^{i} y^{j}$. If $D$ is another bigraded module, the tensor product $C \otimes D$ receives the total bigrading. The identity $P_{C \otimes D}(x, y)=$ $P_{C}(x, y) P_{D}(x, y)$ is easily verified. We obtain a power series in $t$ with the same multiplicative property by setting $Q_{C}(t)=P_{C}(t,-t)$. Observe that

$$
Q_{C}(t)=\sum_{p \geq 0}\left(\sum_{i+j=p}(-1)^{j} \operatorname{dim} C_{i, j}\right) t^{p} .
$$

Let $\mathscr{S}$ be the 1-minimal model; $\mathscr{S}$ is bigraded by $\mathscr{S}_{i, j}=U_{i+j}^{j}$.

LEMMA 3.9. $Q . \gamma(t)=Q(t)$.

PROOF. For each $p$,

$$
\sum_{i+j=p}(-1)^{j} \operatorname{dim} \mathscr{S}_{i, j}=\sum_{j \geq 0}(-1)^{j} \operatorname{dim} U_{p}^{j}=\chi\left(U_{p}\right) .
$$

By definition, $Q(t)=\sum_{p \geq 0} \chi\left(U_{p}\right) t^{p}$, so $Q_{\text {. }}(t)=Q(t)$.

PROOF OF 3.6. Recall $\mathscr{S}=\bigcup_{m>0} \mathscr{S}(m)$, with $\mathscr{S}(0)=\mathbf{R}$ and $\mathscr{S}(m)=$ $\mathscr{S}(m-1) \otimes \Lambda_{1}\left(V_{m}\right)$ for $m \geq 1$. Each $\mathscr{S}(m)$ inherits a bigrading from $\mathscr{S}$. Write $Q_{\text {. }}(m)(t)=Q_{m}(t)$. Then $\lim _{m \rightarrow \infty} Q_{m}(t)=Q(t)$ by 3.9 . We will show by induction that $Q_{m}(t)=\prod_{n=1}^{m}\left(1-t^{n}\right)^{\varphi_{n}}$; the equation $Q(t)=\prod_{n \geq 1}\left(1-t^{n}\right)^{\varphi_{n}}$ of 3.6 will follow by passing to limits on both sides.

First we examine the exterior algebra $\Lambda_{1}\left(V_{m}\right)$, with the bigrading inherited from $\mathscr{S}$. Note that $U_{p}^{j} \cap \Lambda\left(V_{m}\right)=0$ unless $p=m j$, and that $U_{m \jmath}^{j} \cap \Lambda\left(V_{m}\right)=\Lambda^{j}\left(V_{m}\right)$ has dimension $\left(\begin{array}{c}\varphi_{m} \\ j\end{array}\right)$. Thus

$$
Q_{\Lambda\left(V_{m}\right)}(t)=\sum_{j \geq 0}(-1)^{j}\left(\begin{array}{c}
\varphi_{m} \\
j
\end{array}\right) t^{m j}=\left(1-t^{m}\right)^{\varphi_{m}} .
$$

In particular, $Q_{1}(t)=(1-t)^{\varphi_{1}}$, which starts the induction. The inductive step is easily accomplished using $\mathscr{S}(m)=\mathscr{P}(m-1) \otimes \Lambda\left(V_{n}\right)$ and the multiplicative 
property of $Q_{C}(t)$; the bigrading on $\mathscr{S}(m)$ is the total bigrading because both degree and index sum are additive under multiplication. This completes the proof.

We must acknowledge T. Kohno's contributions with regard to the results of this section. The polynomial $Q_{C}(t)$ used in the preceding proof was introduced in [10] - there it is used with a different bigraded algebra to obtain a different power series representation for $\prod_{n>1}\left(1-t^{n}\right)^{\varphi_{n}}$. Our proof of 3.6 is essentially an adaptation of the argument in $[\mathbf{1 0}]$. Also, Kohno proved 3.8 by different methods in $[9]$.

4. Fiber-type arrangements. In [6] it was shown that the LCS formula is satisfied by arrangements of fiber-type. In this section we show that a fiber-type arrangement is necessarily rational $K(\pi, 1)$. Thus the main result of $[\mathbf{6}]$ follows from Corollary 3.8. At this point it is unclear whether we have examples of rational $K(\pi, 1)$ arrangements which are not fiber-type-see remarks following Corollary 3.5. The methods of $[6]$ are mainly group-theoretic; by contrast, the derivation of 3.8 here is essentially a cohomological argument.

Definition 4.1. The arrangement $\mathbf{A}$ in $\mathbf{C}^{l}$ is fiber-type if there is a tower of bundle maps

$$
M=M_{l} \stackrel{p_{l}}{\rightarrow} M_{l-1} \rightarrow \cdots \rightarrow M_{2} \stackrel{p_{2}}{\rightarrow} M_{1}=\mathrm{C}^{*}
$$

such that, for each $k, 2 \leq k \leq l$,

(i) $M_{k}$ is the complement of an arrangement in $\mathbf{C}^{k}$,

(ii) $p_{k}$ is the restriction of a linear map $\mathbf{C}^{k} \rightarrow \mathbf{C}^{k-1}$, and

(iii) the fiber $F_{k}$ of $p_{k}$ is a copy of $\mathbf{C}$ with finitely many points removed.

Terao [16] has shown that $\mathbf{A}$ is fiber-type if and only if the intersection lattice of $\mathbf{A}$ is super-solvable. Observe that the complement of a fiber-type arrangement is a (topological) $K(\pi, 1)$ space - this is a consequence of the long exact homotopy sequences of the fibrations $p_{k}$. Further properties of fiber-type arrangements are tabulated in [5].

To analyze the effect on minimal models of the fibrations $p_{k}$, we will use the Q-completion functor defined in [2]. The actual definition of Q-completion is unenlightening and will be omitted. Interested readers may consult the references.

Let $X$ be a connected complex with minimal model $\mathscr{M}$. The $\mathbf{Q}$-completion $\mathbf{Q}_{\infty} X$ of $X$ can be constructed up to homotopy type from the minimal model $[1,12.2]$. Recall $\pi^{n}(\mathscr{M})$ is the vector space of indecomposable elements of degree $n$ in $\mathscr{M}$. The main property of $\mathbf{Q}$-completion is the following.

Proposition 4.2. Suppose $X$ is connected with finite dimensional Qcohomology groups. Then $\pi_{n}\left(\mathbf{Q}_{\infty} X\right)$ is isomorphic to $\operatorname{Hom}\left(\pi^{n}(\mathscr{M}), \mathbf{Q}\right)$ for all $n \geq 2$.

ProOF. See $[1,12.8($ iii) $)$.

The Q-completion behaves well with respect to nilpotent fibrations.

Proposition 4.3. Let $p: E \rightarrow B$ be a fibration with fiber $F$. Then $\mathbf{Q}_{\infty} p$ : $\mathbf{Q}_{\infty} E \rightarrow \mathbf{Q}_{\infty} B$ is a fibration. If, in addition, $\pi_{1}(B)$ acts nilpotently on $H_{*}(F)$, then the fiber of $\mathbf{Q}_{\infty} p$ is homotopy equivalent to $\mathbf{Q}_{\infty} F$.

Proof. See 5.1 of Chapter II in [2]. 
We refer the reader to Chapter II, 4.1 of [2] for the definition of nilpotent action. For our purposes we need only observe that the trivial action is nilpotent.

For simply-connected spaces, the $\mathbf{Q}$-completion $\mathbf{Q}_{\infty} X$ is homotopy equivalent to the more familiar construction [7] involving localization in the Postnikov decomposition of $X$, the construction originally used by Sullivan. A similar method for constructing $\mathbf{Q}_{\infty} X$ can be used for nilpotent spaces. This leads to the following result, which, together with 4.2 , provides the connection with 2.5 .

PROPOSITION 4.4. If $X$ is a nilpotent space, then $\pi_{n}\left(\mathbf{Q}_{\infty} X\right)$ is isomorphic to $\pi_{n}(X) \otimes \mathbf{Q}$ for each $n \geq 2$.

ProOF. See 3.1 of Chapter V in [2].

In this case $\pi_{1}\left(\mathbf{Q}_{\infty} X\right)$ is the Malcev completion of $\pi_{1}(X)$, and the canonical map $X \rightarrow \mathbf{Q}_{\infty} X$ induces isomorphisms $H_{k}(X ; \mathbf{Z}) \otimes \mathbf{Q} \rightarrow H_{k}\left(\mathbf{Q}_{\infty} X ; \mathbf{Z}\right)$.

The conclusion of 4.4 also holds for some nonnilpotent spaces.

Proposition 4.5. Suppose $X$ is a $K(\pi, 1)$ space with $\pi=\pi_{1}(X)$ a free group of finite rank. Then $\pi_{i}\left(\mathbf{Q}_{\infty} X\right)=0$ for $i \geq 2$.

ProOF. See Chapter IV, 5.3 of $[\mathbf{2}]$.

Let $\mathbf{A}$ be an arrangement in $\mathbf{C}^{l}$ with complement $M$ and minimal model $\mathscr{M}$. Then $\mathbf{A}$ is a rational $K(\pi, 1)$ if $\mathscr{M}$ is generated by elements of degree one. By 4.3 this is equivalent to the asphericity of $\mathbf{Q}_{\infty} M$. This observation leads to the main result of this section.

Proposition 4.6. Suppose $\mathbf{A}$ is a fiber-type arrangement. Then $\mathbf{A}$ is a rational $K(\pi, 1)$.

PROOF. Let

$$
M=M_{l} \stackrel{p_{l}}{\rightarrow} M_{l-1} \rightarrow \cdots \rightarrow M_{2} \stackrel{p_{2}}{\rightarrow} M_{1}=\mathbf{C}^{*}
$$

be the tower of fibrations of Definition 4.1. We will show $\mathbf{Q}_{\infty} M_{k}$ is aspherical for each $k$ using induction on $k$. For $k=1$ this is a special case of 4.5. For $k \geq 2$ we consider the fibration $p_{k}: M_{k} \rightarrow M_{k-1}$ with fiber $F_{k}$. The crucial observation here is that $\pi_{1}\left(M_{k-1}\right)$ acts trivially on $H_{*}\left(F_{k}\right)$. This was proved in [6]. Then, by 4.3 , we have a fibration $\mathbf{Q}_{\infty} p_{k}: \mathbf{Q}_{\infty} M_{k} \rightarrow \mathbf{Q}_{\infty} M_{k-1}$ with fiber homotopy equivalent to $\mathbf{Q}_{\infty} F_{k}$. By the inductive hypothesis $\mathbf{Q}_{\infty} M_{k-1}$ is aspherical, and $\mathbf{Q}_{\infty} F_{k}$ is aspherical by 4.5 (recall $F_{k}$ is a plane with finitely many punctures). The long exact homotopy sequence of the fibration $\mathbf{Q}_{\infty} p_{k}$ yields $\pi_{n}\left(\mathbf{Q}_{\infty} M_{k}\right)=0$ for $n \geq 2$. The result follows by induction.

COROLlary $4.7[\mathbf{6}]$. Suppose $\mathbf{A}$ is a fiber-type arrangement. Then A satisfies the LCS formula.

5. Algebraically 2-determined arrangements. In this section we derive a simple necessary condition for an arrangement to be 2-determined (recall Definition 3.2). This result allows us to exhibit a counterexample for the converse of 3.8. Specifically, we show that the reflection arrangement of type $D_{4}$ is not 2-determined, and therefore not rational $K(\pi, 1)$. The LCS formula has been verified for this arrangement by Kohno [8]. 
Proposition 5.1. Let $\mathbf{A}$ be an arrangement in $\mathbf{C}^{l}$. Suppose $\mathbf{A}$ contains hyperplanes $H_{i}, 1 \leq i \leq k+1$, satisfying the following conditions:

(i) $\bigcap_{i=1}^{k+1} H_{i}$ has codimension $k$ in $\mathbf{C}^{l}$, and

(ii) for $1 \leq i<j \leq k, H_{i} \cap H_{j}$ is contained in no other hyperplane of $\mathbf{A}$.

Then $\mathbf{A}$ is not 2-determined.

PROOF. Let $e_{i}$ be the generator of $\mathscr{E}$ corresponding to $H_{i}$ for $1 \leq i \leq k+1$. By (i), $\partial\left(e_{1} \cdots e_{k+1}\right)$ is an element of the relation ideal $\mathscr{I}$. We claim that this element is not contained in $\mathscr{J}$, the ideal generated by $\mathscr{J}^{2}$. To see this, observe that (ii) implies that no monomial in $\mathscr{I}^{2}$ involves two of the $e_{i}$ for $1 \leq i \leq k$. Therefore $e_{1} \cdots e_{k}$ cannot be an element of $\mathscr{J}^{k}=\mathscr{E}^{k-2} \wedge \mathscr{I}^{2}$. But $\pm e_{1} \cdots e_{k}$ is the last term in $\partial\left(e_{1} \cdots e_{k+1}\right)$. Since $\mathscr{E}$ is free, the claim follows. Thus $\mathscr{J} \neq \mathscr{I}$, and therefore A is not 2-determined.

COROLlARY 5.2. Under the hypothesis of 5.1, $\mathbf{A}$ is not a rational $K(\pi, 1)$ arrangement.

ProOF. This is immediate from 5.1 and 3.5.

Let us return to Example 2.19. The hyperplanes $x=y, x=-y, z=0$, and $x=z$ satisfy the conditions of 5.1. Therefore the arrangement of 2.19 is not rational $K(\pi, 1)$, though it is a topological $K(\pi, 1)$. This was noted in $\S 3$, using the fact that the LCS formula fails. Chronologically, Corollary 5.2 was discovered prior to Corollary 3.8.

EXAMPLE 5.3. Let $\mathbf{A}$ be the arrangement in $\mathbf{C}^{4}$ consisting of the 12 hyperplanes $z_{i}= \pm z_{j}, 1 \leq i<j \leq 4$. This is the arrangement of reflecting hyperplanes for the Weyl group $D_{4}$. The hyperplanes $z_{1}=z_{2}, z_{1}=-z_{2}, z_{3}=z_{4}, z_{3}=-z_{4}$, and $z_{1}=z_{4}$ satisfy the conditions of 5.1. Therefore this arrangement is not rational $K(\pi, 1)$. But the LCS formula holds in this case. Indeed, Kohno [8] has established the LCS formula for all the $D_{l}$ arrangements. Thus the implication "LCS implies rational $K(\pi, 1)$ " is false.

\section{REFERENCES}

1. A. Bousfield and V. Guggenheim, On PL DeRham theory and rational homotopy type, Mem. Amer. Math. Soc., vol. 8, No. 179, Amer. Math. Soc., Providence, R.I., 1976.

2. A. Bousfield and D. Kan, Homotopy limits, completions, and localizations, Lecture Notes in Math., vol. 304, Springer-Verlag, Berlin, 1972.

3. E. Brieskorn, Sur les groupes de tresses (d'aprés V. I. Arnol'd), Séminaire Bourbaki $24^{\mathrm{e}}$ année 1971/72, Lecture Notes in Math., vol. 317, Springer-Verlag, Berlin, 1973.

4. P. Deligne, Les immeubles des groupes de tresses généralises, Invent. Math. 17 (1972), 273-302.

5. M. Falk and R. Randell, On the homotopy theory of arrangements, Complex Analytic Singularities, Adv. Studies in Pure Math., no. 8, North-Holland, Amsterdam, 1986, pp. 101-124.

6. __ The lower central series of a fiber-type arrangement, Invent. Math. 82 (1985), 77-88.

7. P. Griffiths and J. Morgan, Rational homotopy theory and differential forms, Birkhäuser, Boston, Mass. and Basil, 1981.

8. T. Kohno, Poincaré series of the Malcev completion of generalized pure braid groups, preprint.

9. __ Rational $K(\pi, 1)$ arrangements satisfy the LCS formula, preprint.

10. $\ldots$, Séries de Poincaré-Koszul associée aux groupes de tresse pure, Invent. Math. 82 (1985), 57-75.

11. W. Magnus, A. Karrass, and D. Solitar, Combinatorial group theory, Wiley, New York, 1966.

12. J. Morgan, The algebraic topology of smooth algebraic varieties, Inst. Hautes Étude Sci. Publ. Math. 48 (1978), 137-204. 
13. P. Orlik and L. Solomon, Combinatorics and topology of complements of hyperplanes, Invent. Math. 56 (1980), 167-189.

14. D. Sullivan, Infinitesimal computations in topology, Inst. Hautes Étude Sci. Publ. Math. 47 (1977), 269-331.

15. H. Terao, Generalized exponents of a free arrangement of hyperplanes and Shephard-ToddBrieskorn formula, Invent. Math. 63 (1981), 159-179.

16. __ Modular elements of lattices and topological fibrations, Adv. in Math. 62 (1986), 135-154.

Department of Mathematics, The University of Iowa, Iowa City, IOWA 52242

Department of Mathematics, Northern Arizona University, Flagstaff, ARIZONA 86004 